\title{
VARIOUS APPROACHES IN CLASSIFICATION OF TECHNICAL PROCESSES
}

\author{
Petr Bečváŕ, Luděk Müller \\ Department of Cybernetics, Faculty of Applied Sciences, University of West Bohemia, \\ Czech Republic \\ becvar@kky.zcu.cz \\ muller@kky.zcu.cz
}

\begin{abstract}
Within nuclear reactor diagnostics, it is necessary to automatically recognise the operations which have occurred. These operations (called transient events) have to be recognised from the measured data stream. Because it is not possible to determine the boundaries between the transient events, the entire sequence of events has to be recognised. Also, the duration of the similar processes of the same class differs in different realisations. Several methods have been used to solve this problem. Three of them are discussed in this article: human recognition, a program based on a pattern recognition method (so-called Hidden Markov Models) and a program using pattern recognition for generation of hypotheses and a rule based expert system for hypotheses testing.
\end{abstract}

\section{INTRODUCTION}

This paper deals with principles of technical process classification which are described in the context of an industrial application. Process classification is frequently applied when performing technical diagnostics. When a human expert is presented a data stream graph measured during the operation of a device, he must recognise events (processes) that occurred during this operation. While the human expert can solve this task intuitively, it is very demanding for the artificial intelligence to process the recognition. The process classification task we describe is part of a larger on-line diagnostic system for monitoring the residual life of a nuclear reactor pressure vessel shell.

\section{THE PRIMARY PROBLEM}

It is a very demanding task to determine residual life. The finite element method (FEM) is used to compute temperature and stress values at critical points of the shell 
material. These values are evaluated by FEM from measured temperatures given by a set of thermocouples. The solution time required by FEM is very long and for this reason a more sophisticated approach for on-line evaluation is necessary.

To reduce computing time, it would be convenient to pre-process as much data as possible. The pre-computed result of the evaluation of the standard transient event pattern can be used instead of the result of the FEM evaluation of on-line measured transient events. Because of strict rules of operation within the nuclear power plant (Sik, 1993), there is a limited number of different possible transient events

As result of this, the task is now much more straightforward - we have to convert all permanently measured data into a sequence of transient events. This sequence is then replaced by a sequence of pre-computed FEM. A robust algorithm is then used to evaluate the residual life of the vessel.

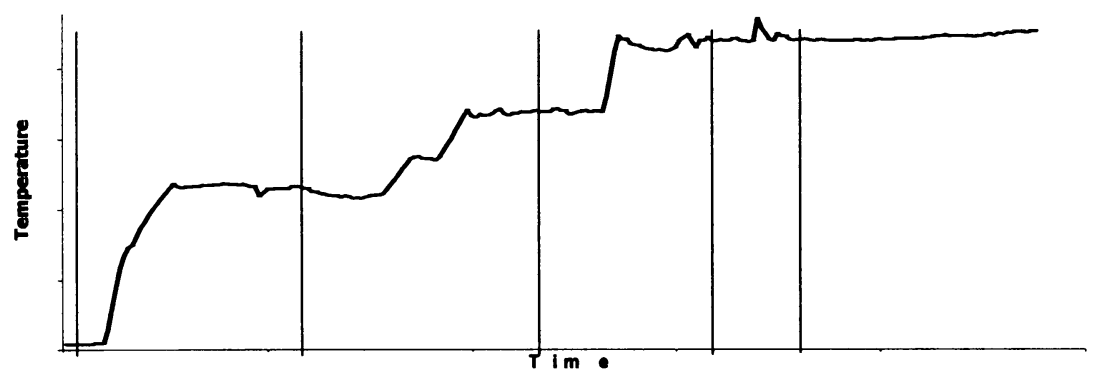

Figure 1 - Example of a sequence of transient events

This paper presents several approaches to the classification of measured data into a sequence of predefined transient events. Figure 1 shows a sample temperature graph during a sequence of transient events, where lines separate the different events. It is evident how difficult it is to find boundaries between the events in the sequence.

At least three approaches can be used to resolve the task.

1. Human classification supported by various levels of computer support

2. Computer program based on pattern recognition with human supervision

3. Hybrid system based on hypothesis generation and verification

Each of these possibilities has its advantages and disadvantages. Each possibility will be briefly discussed and our experience with the $2^{\text {nd }}$ possibility will then be presented. Finally, we offer an explanation of why the implemented system should be improved, as well as a brief description of the technology used for this improvement

\subsection{Human classification}

Human classification has been used until now and it gives satisfactory results. The main reason for applying an automated system, is the fact that each human being may be influenced by his respective point of view. Objectivity and reliability are main advantages of a computer program although human supervision is necessary for every system. 


\subsection{Automated classification based on the pattern recognition}

Searching for the best sequence of patterns assigned to an unbroken signal is a standard problem in speech recognition which can be solved by a technique based on so-called Hidden Markov Models (HMM) and a decoding procedure that uses dynamic programming.

HMM's are the templates of certain transient events that and encode the expert knowledge used for recognition. These templates are composed by a system designer based on expert knowledge.

It should be noted that problems can still occur even if the system has been designed and tested with the satisfactory results.

\subsection{Hybrid system}

A method should be developed to eliminate the problems that can arise with HMMs while retaining their fundamental advantages. For example, an approach based on blackboard control which combines HMM-based knowledge sources with rule-based knowledge sources seems to be highly advisable.

The HMM-based or neural net based knowledge sources can work as hypothesis generators. Their results are verified at a higher level by the rule-based knowledge sources and combined into sequences of transient events. Any type of knowledge can be included in such a system.

\section{THEORY OF HIDDEN MARKOV MODELS}

Hidden Markov Models are very useful in process classification as they are used in both approaches to automated event recognition.

\subsection{Isolated event recognition}

Let each transient event be represented by a sequence of vectors or observations $\boldsymbol{O}$, defined as $\boldsymbol{O}=\left\{\boldsymbol{a}_{1}, \boldsymbol{o}_{2}, \mathrm{~K}, \boldsymbol{o}_{t}, \mathrm{~K}, \boldsymbol{o}_{T}\right\}$ where $\boldsymbol{o}_{t}$ is the vector observed at time $t$. The isolated transient event recognition problem can be regarded as a problem of computing

$$
\underset{i}{\operatorname{argmax}}\left\{P\left(u_{i} \mid \boldsymbol{O}\right)\right\}
$$

where $w_{i}$ is the $i$-th transient event. This probability is not directly computable but using Bayes' rule gives

$$
P\left(w_{i} \mid \boldsymbol{O}\right)=\frac{P\left(\boldsymbol{O} \mid w_{i}\right) \cdot P\left(w_{i}\right)}{P(\boldsymbol{O})}
$$


Thus, for a given set of prior probabilities $P\left(w_{i}\right)$, the most probable transient event depends only on the likelihood $P\left(O \mid w_{i}\right)$. By applying the maximum likelihood estimation technique, the problem of estimating the class of conditional observation densities $P\left(\boldsymbol{O} \mid w_{i}\right)$ can be replaced by the much simpler task of estimating the Markov model parameters.

In HMM-based recognition, it is assumed that the sequence of observed vectors corresponding to each transient event is generated by a Markov model. A Markov model is a finite state machine which changes state once every time unit and each time $t$ that a state $j$ is entered, a vector $o_{t}$ is generated from the probability density $b_{f}\left(o_{t}\right)$. Furthermore, the transition from state $i$ to state $j$ is also probabilistic and is governed by the discrete probability $a_{i j}$. Fig. 2 shows an example of this process where the six state model moves through the state sequence $X=1,1,3,4$ in order to generate the sequence $o_{1}$ to $o_{4}$ (Young, 1999).

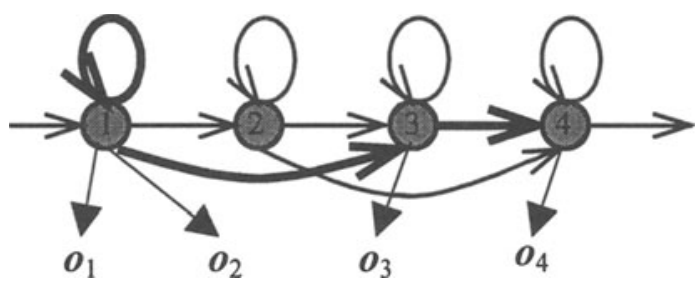

Figure 2 - The Markov generation model

The joint probability that $O$ is generated by the model $M$ moving through the state sequence $X$ is calculated simply as the product of the transition probabilities and the output probabilities.

However, in practice, only the observation sequence $O$ is known and the underlying state sequence $X$ is hidden. This is why it is called a Hidden Markov Model.

Given that $X$ is unknown, the required likelihood is computed by summing all possible state sequences $X=x(1), x(2), x(3), \ldots, x(T)$, that is

$$
P(\boldsymbol{O} \mid M)=\sum_{\boldsymbol{X}} a_{x(0) x(1)} \prod_{t=1}^{T} b_{x(t)}\left(o_{t}\right) a_{x(t) x(t+1)}
$$

where $x(0)$ is constrained to be the model entry state and $x(T+1)$ is constrained to be the model exit state.

As an alternative to equation 3 , the likelihood can be approximated by only considering the most likely state sequence, that is

$$
\bar{P}(\boldsymbol{O} \mid M)=\max _{X}\left\{a_{x(0) x(1)} \prod_{t=1}^{T} b_{x(t)}\left(\boldsymbol{o}_{t}\right) a_{x(t) x(t+1)}\right\}
$$

Although the direct computation of equations 3 and 4 is not tractable, simple recursive procedures exist which allow both quantities to be calculated very 
efficiently. All this, of course, assumes that the parameters $\left\{a_{i j}\right\}$ and $\left\{b_{j}\left(o_{t}\right)\right\}$ are known for each model $M_{i}$.

HMMs are designed primarily for modelling continuous parameters using continuous density multivariate output distributions. Therefore output distributions $b_{j}\left(\boldsymbol{o}_{t}\right)$ are represented by Gaussian densities.

\subsection{Recognition and Viterbi decoding}

In practice it is preferable to base recognition on the maximum likelihood of the state sequence since this generalises easily to the case of the transient event sequence. This likelihood is computed using essentially the following algorithm.

For a given model $M$, let $\phi_{j}(t)$ represent the maximum likelihood of observing vectors $o_{1}$ to $o_{t}$ and being in state $j$ at time $t$ (Young, 1997). This partial likelihood can be computed efficiently using the following recursion:

$\phi_{j}(t)=\max _{i}\left\{\phi_{i}(t-1) \cdot a_{i j}\right\} \cdot b_{j}\left(o_{t}\right)$

where

$\phi_{1}(0)=1$
$\phi_{j}(0)=0, \quad 1<j \leq N$

The maximum likelihood $P\left(O \mid w_{i}\right)$ is given by

$P\left(\boldsymbol{O} \mid w_{i}\right)=\phi_{N}(T)=\max _{i}\left\{\phi_{i}(T) \cdot a_{i N}\right\} \cdot b_{N}\left(\boldsymbol{o}_{T}\right)$

This recursion forms the basis of the so-called Viterbi algorithm. At time $t$, each partial probability $\phi_{i}(t-1)$ is known for all states $i$, hence equation 5 can be used to compute $\phi_{i}(t)$ thereby updating the partial probabilities by one time vector.

\subsection{Connecting models into sequences}

The previous section deals with recognition of a single transient event. To recognise whole sequences we can exploit another advantage of HMMs - the possibility of connecting models together and using the Viterbi algorithm to evaluate connected models together as one large composed HMM.

The exit state of the model $i$ is connected with the entry states of the models of the transient events which can follow the transient event $i$. Part of the prior expert knowledge is a strict definition of all possible transient event sequences. These data can be stored in a single model-to-model transition probability matrix $\boldsymbol{C}$, where $c_{p, k}$ is the discrete probability that the transient event $k$ follows the transient event $p$. 


\section{COMPUTER PROGRAM BASED ON PATTERN RECOGNITION WITH HUMAN SUPERVISION}

As mentioned above, a system based on this approach has already been developed and tested. This section briefly explains parts of the system and the most serious weaknesses that were encountered during design. The system is implemented in $\mathrm{C}++$ and it stringently separates program code and expert knowledge.

The duration of a transient event is from 2.5 minutes up to 28 hours. Because the minimum length of the model is 2 states, the maximum length of the HMM can be more then 1000 states. We can utilise the given definitions of the transient events and set the HMM parameters according to our prior knowledge (Šik, 1993).

One serious problem arose, when a short stable state appeared in the event sequence. The algorithm tries to assign the nearest event to it and a sequence of short increasing and decreasing events appears on the output. These short events represent fast changes of the temperature of the reactor and strongly influence the residual life. It was therefore necessary to add a model of the short stable period. This model also includes a model of the short glitch and several other measurement errors (Bečár, 1999).

Several disadvantages of the approach appeared during work:

- The system looks for the sequence of patterns which best matches to an input signal. If there is a part of the signal that does not correspond to any pattern, the program returns a nonsense output.

- Some events (like a short glitch) can not be modelled using HMM technology. A special model must be designed and implemented as a $\mathrm{C}++$ code, which decreases the variability of the system.

- The physical model and the model of the control rules are simplified into one model-to-model transition probability matrix. This method allows only shallow knowledge of the model to be represented, which was found inadequate.

\section{HYBRID SYSTEM}

The two most important approaches on which the hybrid system is based are: the blackboard architecture and the HMM.

The HMM's are used as described above, only with a small modification. In addition to equation 6 for every time $t$ we set:

$\phi_{1}(t)=1 \quad \forall t$

The model begins evaluation at every time step and it evaluates all time periods under the same condition. The value $\phi_{N}(t)$ gives the probability that a previous data corresponds to the investigated model. At each time $t_{h}$

$t_{h}=\underset{t \in I}{\operatorname{argmax}}\left(\phi_{N}(t)\right) \quad I=<t_{h}-d t, t_{h}+d t>$ 
for the given $d t$ a hypothesis is generated that the template has been recognised. The certainty factor of this hypothesis is equal to $\phi_{N}\left(t_{h}\right)$. The high-level knowledge sources have to verify or reject this hypothesis later.

\subsection{Blackboard architecture}

Blackboard style (Pfleger, 1998) refers to a particular way of organising high level computing systems. In its most basic form, the blackboard style separates a system into a central, globally-accessible data structure, typically called the blackboard, and a number of independent computational modules, often called knowledge sources, or KSs. The knowledge sources perform computations based on the contents of the blackboard in order to change the blackboard.

Not all KSs are appropriate for execution at any given time. Instead, only a certain subset of all available KSs is enabled at any time. Thus, each KS has two subcomponents, one that determines whether or not the $\mathrm{KS}$ is enabled, called the KS precondition, and one that encodes the computation to be performed by the KS when executed, called the KS body.

This brings us to the third important component of a blackboard system, control. Only a single KS can be executed at a time, necessitating a method of choosing a single KS for execution. This problem is known as the control problem, and the method used to solve it is known as the control strategy. Blackboard systems differ greatly in their individual control strategies.

The blackboard style has many advantages:

- The partitioning into separate knowledge sources creates modularity, which allows KSs to be modified, added, or removed from the system easily.

- The basic control cycle of the blackboard style allows it to simultaneously support multiple reasoning strategies, and to switch flexibly between them.

- The automatic enabling of KSs also provides a natural type of opportunism, facilitating automatic exploitation of beneficial situations, or an automatic mechanism for reaction to urgent problems.

As with any organisational style, there are a few disadvantages to the blackboard approach:

- The shared aspect of the blackboard data structures creates a heavy dependence on the choice of representation for data there.

- Since there is no data hiding, integrity and consistency constraints must be carefully maintained by every $\mathrm{KS}$ that manipulates that data.

- In general, KSs are completely independent. This means that opportunities for reuse of KS components are wasted.

\subsection{Higher-level knowledge sources}

The higher-level knowledge source is a name used for other programs which have to be implemented and included into the system in order to verify hypotheses and to construct a general hypothesis.

The best strategy is to implement them as a rule-based expert system. The advantage of this approach is the universality of the rule-based system Such a system 
must be encapsulated by a universal interface that is able to communicate via the blackboard.

Knowledge about a physical model, the system history and control restrictions can easily be included in such knowledge sources but not in the transition probability matrix. The blackboard system does not rule out implementations of the KSs using alternative approaches like neural nets, provided they are suitable for the task

\section{CONCLUSION}

As is evident from the previous text, many methods can be used to solve the process classification task. As is typical for artificial intelligence tasks, the program must include considerable expert knowledge in order to achieve the standard of a human expert.

A system based only on Hidden Markov Model recognition can solve the problem very effectively and with sufficient reliability. The quality of models used is crucial because the model encodes almost all knowledge used for evaluation. Here appears the main problem: the use of any other approach to control the results of the recogniser is complicated.

A more sophisticated system is currently being developed to eliminate the previously mentioned disadvantage. This system should generate a certain number of the hypotheses and combine them into a general solution supported by knowledge about the physical model. This method seeks to retain the main advantage of HMMs (the ability to find a given template even in a distorted continuous signal) and to support it by other methods overcoming the dependency on only one knowledge source.

\subsection{Acknowledgments}

This work was supported by projects No. VS97159 and No. MSM23520004 of the Ministry of Education

\section{REFERENCES}

1. Bečváł, Muller, Psutka. Classification of Transient Events of Nuclear Reactor Using Hidden Markov Model. First International Conference on Advanced Engineering Design, Praha, 1999.

2. Pfleger K,Hayes-Roth B. An Introduction to Blackboard-Style Systems Organization. Knowledge Systems Laboratory, Stanford, Technical Report, 1998.

3. Šik, Hulínová, Kubica. Provozní a płechodové režimy reaktorového bloku EDU. ŠKODA, Jaderné strojírenství, Plzeñ, Technický servis, odbor výpocty, Plzeñ, 1993.

4. Young S, Bloothooft G. Corpus-Based Methods in Language and Speech Processing. Kluwer Academic Publishers, 1997.

5. Young S, Kershaw D at al. The HTK Book (for HTK Version 2.2). Entropic Ltd, 1999. 